around this time adds significant complexity. Sheffield has a seamless adolescent rheumatology service spanning both paediatric and adult hospitals, aiming to provide developmentally appropriate healthcare (DAH). To exemplify the benefits of this approach, we present two adolescents with the rare combination of large vessel vasculitis and inflammatory bowel disease. Case A was diagnosed in the Children's hospital and B in adult services, within 1 month of each other. Within the seamless service we were able to coordinate a patient-centred approach both for complex medicines management and ongoing clinical care.

A: 17 year-old male with complex inflammatory bowel disease since age 2. Treated with oral steroids with poor response to multiple DMARDs, biologics and elemental nutrition. Health professionals doubted good adherence and he needed extensive input. Vedolizumab was started in 2019 with improvement of gut symptoms but subsequent investigation of neck pain revealed stenotic large vessel vasculitis (LVV) and a diagnosis of Takayasu arteritis was made.

B: 17 year-old male with ulcerative colitis, diagnosed aged 14. Treated with infliximab and azathioprine when transferred to adult gastroenterology services. Atypical symptoms developed in 2019 and chest CT/PET-CT confirmed LVV (Takayasu arteritis).

LVV is a rare, life-threatening condition. Well-established team working enabled sharing of paediatric and adult rheumatology and gastroenterology experience. This enabled rational, tailored treatment plans to be devised through shared professional expertise - A has stopped vedolizumab and started tocilizumab, B has started adalimumab and both started methotrexate.

LVV needs lifetime monitoring. Planning for that monitoring (imaging, serology, biopsychosocial care) in adult services is therefore an important part of initial management. A and B are at different developmental stages. Our service enables DAH with A remaining in paediatrics, but with care planned to facilitate transition and ongoing management in adult services, B continues in adult services with the benefit of paediatric experience in managing Takayasu arteritis.

'Adolescent responsive services' are key in developmentally appropriate healthcarecare. The Sheffield Seamless Adolescent Rheumatology service is an example of this in practice.

\section{G537(P) VITAMIN D PREPARATIONS: WHAT'S IN THE BOTTLE?}

$1,2 \mathrm{M}$ Wan, ${ }^{2} \mathrm{~A}$ Patel, $2,3 \mathrm{JP}$ Patel, ${ }^{4} \mathrm{G}$ Rait, ${ }^{2} \mathrm{~S}$ Jones, ${ }^{5} \mathrm{R}$ Shroff. ${ }^{1}$ Evelina Pharmacy, Guy's and St Thomas' NHS FT, London,UK; ${ }^{2}$ Institute of Pharmaceutical Science, King's College London, London, UK; ${ }^{3}$ Department of Haematological Medicine, King's College Hospital NHS FT, London, UK; ${ }^{4}$ Research Department of Primary Care and Population Health, University of College London, London, UK; ${ }^{5}$ Renal Unit, Great Ormond Street Hospital NHS FT, London, UK

\subsection{6/archdischild-2020-rcpch.455}

Aims The UK incidence of vitamin D prescribing in children has increased by 26-fold in recent years. Public Health England recommends that children over 1 year take a daily vitamin D supplement. But the availability of over 200 different vitamin D products can be confusing for parents and clinicians. Our study aimed to assess the usage of licensed and unlicensed vitamin D only (VDO) preparations across primary care in England, and to compare measured and labelled vitamin D content of VDO preparations marketed in England.

Methods

- Analysis of the vitamin D content of randomly selected VDO preparations using reversed-phase high performance liquid chromatography.

- Retrospective trend analysis of prescription reimbursement data for VDO prescriptions from 2008 to 2018.

Results Licensed and unlicensed VDO preparations were available in a wide range of dose strengths from 400 to 50,000 IU. The number of licensed VDO preparations increased from 4 to 32 between 2008 and 2018, along with an increase in the proportion of VDO prescriptions fulfilled by licensed preparations. However, prescriptions of unlicensed preparations remained high and accounted for $42 \%$ of the prescription items in 2018. The 11 unlicensed preparations analysed had vitamin $\mathrm{D}$ concentrations ranging from $41.2 \pm 10.6 \%$ to $165.3 \pm 17.8 \%$ of the declared content, with only one meeting the acceptable criteria of $90-125 \%$. The 2 licensed preparations met the required standards. There was no association between the preparation dose strength and the magnitude of percentage difference between measured and labelled contents $(\mathrm{r}=0.41, p=0.17)$. Unlicensed liquid preparations in dropper bottles showed the greatest inter-sample variability suggesting there may be a lack of uniformity in drop volume delivered which could lead to dose variability.

Conclusion Despite the increasing availability of licensed preparations with assured quality, use of unlicensed preparations to fulfil VDO prescriptions has continued in primary care in England. Unlicensed VDO preparations marketed showed wide variations between measured and declared vitamin D contents. Younger children who are more vulnerable to harm are thus exposed to unnecessary risks of under- and oversupplementation.

\section{G538(P) YOUTH VIOLENCE: HOW CAN WE INTERVENE?}

${ }^{1,2} \mathrm{~S}$ Jen, ${ }^{1} \mathrm{~N}$ Quah Qin Xian, ${ }^{1} \mathrm{~K}$ Sullivan, ${ }^{1} \mathrm{~K}$ Malbon. ${ }^{1}$ Department of Paediatrics, Imperial College Healthcare Trust, London, UK; ${ }^{2}$ University College London Hospitals NHS FT, London, UK

\subsection{6/archdischild-2020-rcpch.456}

Introduction Interpersonal violence involving sharp instruments or firearms continues to rise in the UK. Recent evidence indicates an association between social deprivation and violence against youths, emphasising the importance of addressing psychosocial issues early in adolescence through appropriate assessment and delivery of psychosocial care. The HEEADSSS assessment is a valuable tool in helping clinicians identify areas of psychosocial concern. This study aims to identify the epidemiology of such assaults in London presenting to a local trauma centre, assess whether residence in a high-depravity area increases the risk of violent injury, and determine whether further psychosocial steps can be taken to reduce risk of reoccurrence in these individuals.

Methods Data from the emergency department of a major trauma centre in West London was retrospectively reviewed in those $<25$ years presenting in 2018 with injuries arising from assault with weapon. Gender, age, home postcode, data from 